\title{
Emission lines in GRBs constrain the total energy reservoir
}

\author{
G. Ghisellini ${ }^{1}$, D. Lazzati ${ }^{2}$, E. Rossi ${ }^{2}$, and M. J. Rees ${ }^{2}$
}

1 Osservatorio Astronomico di Brera, via Bianchi 46, 23807 Merate, Italy

2 Institute of Astronomy, Madingley Road, CB3 0HA Cambridge, UK

Received 19 April 2002 / Accepted 14 May 2002

\begin{abstract}
The emission features observed in the X-ray afterglow of Gamma Ray Bursts are extremely powerful. Since they last at least for several hours, they imply energies of the order of $10^{49}$ ergs. This in turn implies that the energy contained in the illuminating continuum thought to be responsible of the line production must exceed $10^{51}$ ergs. This is a strong lower limit to the energy reservoir of Gamma Ray Bursts, which is independent of collimation and beaming, and bears important consequences on the possible collimation of the fireball radiation and the density of the medium surrounding the burst.
\end{abstract}

Key words. gamma rays: bursts - line: formation - radiation mechanisms: general

\section{Introduction}

The discovery that Gamma Ray Bursts (GRBs) are cosmological implies large luminosities, but the exact value of the radiated luminosities and the kinetic power of the fireball originating the emission are still uncertain by a large factor, since we do not know if the emission is isotropic or if it is instead collimated in a cone. The main tool to estimate the degree of collimation of the emission has been, so far, the presence of an achromatic break in the lightcurves of the afterglows. This is interpreted as due to the deceleration of the fireball, whose bulk Lorentz factor $\Gamma$ becomes smaller than the inverse of the jet opening angle $\theta_{\mathrm{j}}$ (see e.g. Rhoads 1999). This allows to estimate $\theta_{\mathrm{j}}$ and to obtain the "true" values of the emitted energy. Most notably, in this respect, is the finding of Frail et al. (2001) who found a remarkable "clustering" of the fireball energy, once they are corrected by the estimate of their degree of collimation. Since the total power and energy are obviously the main parameters for the construction of any model, the importance of these estimates is obvious. These in turn are based on a number of assumptions, such as the density of the matter surrounding the burst site, which is responsible for the deceleration of the fireball, and on the key observation of the presence of an achromatic break in the light curve of the afterglow.

Independent estimates of the "true" energy, or even limits on it, are called for. In this paper we point out that the observed emission features observed in the X-ray afterglow spectra of several bursts can indeed put a firm lower limit to the emitted luminosity of GRBs. This limit is at the same time simple, independent of the degree of

Send offprint requests to: G. Ghisellini, e-mail: gabriele@merate.mi.astro.it collimation of the burst radiation, and independent of the density of the surrounding medium.

\section{Energy of the observed emission features}

Table 1 reports the main information obtained for the five bursts for which emission features have been detected so far. For GRB 970828 the redshift listed in Table 1 is uncertain since this burst did not have a standard optical or radio afterglow and therefore the identification of the host galaxy should be regarded as tentative. For GRB 000214 the value of redshift listed in Table 1 is even more uncertain, since it corresponds to assume that the feature observed in $\mathrm{X}$-rays is a iron $6.97 \mathrm{keV}$ emission line.

The significance of each of the line detections, so far, is at the level of only $3-4 \sigma$, with the iron line of GRB 991216 (observed by Chandra) being the best evidence (see references listed in Table 1). Thus their reality is still under debate, and the issue will likely be settled only with future observations, even if the fact that they have been "observed" so far by the BeppoSAX, ASCA, Chandra and XMM-Newton satellites brings some weight to their reality.

The line luminosities listed in Table 1 are derived under the assumption of isotropic emission (but see below); the start time $t_{\mathrm{s}}$ corresponds to the beginning of the $\mathrm{X}$-ray observation which led to the discovery of the emission feature, while the end time $t_{\mathrm{e}}$ corresponds to the time for which either the line was not detected any longer or to the ending of the observations. We give, in Table 1, two values of the total line energy: the first assumes that the line existed only for the time interval $\left(t_{\mathrm{e}}-t_{\mathrm{s}}\right)$ ("short lived" line), while the second assumes that the line remained constant in flux for $\left(t_{\mathrm{e}}-0\right)$ ("long lived" line). Indications in favor of a line of constant flux come from GRB 000214 (Antonelli et al. 2000), while for GRB 970508 and 
Table 1. Luminosities and energies are calculated assuming $H_{0}=65 \mathrm{~km} \mathrm{~s}^{-1} \mathrm{Mpc}^{-1}, \Omega_{\Lambda}=0.7$ and $\Omega_{\mathrm{m}}=0.3$, and assuming that the line emission is isotropic. We use the notation $Q \equiv 10^{x} Q_{x}$. The redshift of GRB 000214 is calculated assuming that the observed line is from $\mathrm{H}$-like iron. The time $t_{\mathrm{s}}$ is the start time of the $\mathrm{X}$-ray afterglow observations; $t_{\mathrm{e}}$ is the time until which the line was visible. $\left(E_{49}^{\text {iso }}\right)^{a}$ corresponds to assuming that the line was constant in flux (and emitted isotropically) in the time interval $\left(t_{\mathrm{e}}-t_{\mathrm{s}}\right) ;\left(E_{49}^{\mathrm{iso}}\right)^{b}$ corresponds to assuming a constant line in the time interval $\left(t_{\mathrm{e}}-0\right)$. These values take into account the cosmological time dilation factor $(1+z)$. The last three columns report the jet opening angle and the corrected energy emitted in $\gamma$-rays as calculated by Frail et al. (2001) and our estimated range of $E_{\gamma}$ using $\eta_{\text {line }}=0.02$ for all bursts but GRB 011211, for which we used $\eta_{\text {line }}=0.05$. References: A00: Antonelli et al. (2000); P99: Piro et al. (1999); P00: Piro et al. (2000); R02: Reeves et al. (2002); Y99: Yoshida et al. (1999).

\begin{tabular}{|c|c|c|c|c|c|c|c|c|c|c|c|}
\hline GRB & $z_{\mathrm{opt}}$ & $\begin{array}{l}F_{-14}^{\text {line }} \\
\operatorname{erg~s}^{-1} \mathrm{~cm}^{-2}\end{array}$ & $\begin{array}{l}\epsilon^{\text {line }} \\
\mathrm{keV}\end{array}$ & $\begin{array}{l}t_{\mathrm{s}}-t_{\mathrm{e}} \\
\mathrm{h}\end{array}$ & $\begin{array}{l}L_{44}^{\text {iso }} \\
\operatorname{erg~s}^{-1}\end{array}$ & $\begin{array}{l}\left(E_{49}^{\text {iso }}\right)^{a} \\
\text { erg }\end{array}$ & $\begin{array}{l}\left(E_{49}^{\text {iso }}\right)^{b} \\
\text { erg }\end{array}$ & Ref. & $\begin{array}{l}\theta_{\mathrm{j}}^{\mathrm{F} 01} \\
\mathrm{deg}\end{array}$ & $\begin{array}{l}E_{\gamma, 51}^{\mathrm{FO} 1} \\
\mathrm{erg}\end{array}$ & $\begin{array}{l}E_{\gamma, 51} \\
\text { erg }\end{array}$ \\
\hline 970508 & 0.835 & $30 \pm 10$ & $3.4 \pm 0.3$ & $6-16$ & $12 \pm 4$ & $2.25 \pm 0.8$ & $3.6 \pm 1.3$ & P99 & 16.7 & 0.23 & $11-18$ \\
\hline 991216 & 1.02 & $17 \pm 5$ & $3.5 \pm 0.06$ & $37-40$ & $11 \pm 3$ & $0.6 \pm 0.2$ & $7.7 \pm 2.3$ & P00 & 2.9 & 0.69 & $3-38$ \\
\hline 000214 & $0.46 ?$ & $6.7 \pm 2.2$ & $4.7 \pm 0.2$ & $12-41$ & $0.6 \pm 0.2$ & $0.4 \pm 0.14$ & $0.6 \pm 0.3$ & A00 & - & - & $2-3$ \\
\hline 011211 & 2.14 & $0.8 \pm 0.5$ & $\mathrm{Mg}$ & $11-12.4$ & $3 \pm 2$ & $0.05 \pm 0.04$ & $0.4 \pm 0.3$ & R02 & - & - & \\
\hline 011211 & 2.14 & $0.7 \pm 0.25$ & $\mathrm{Ar}$ & $11-12.4$ & $2.6 \pm 1$ & $0.04 \pm 0.016$ & $0.4 \pm 0.14$ & R02 & - & - & \\
\hline 011211 & 2.14 & $0.44 \pm 0.22$ & $\mathrm{Ca}$ & $11-12.4$ & $1.7 \pm 0.9$ & $0.03 \pm 0.015$ & $0.25 \pm 0.12$ & R02 & - & - & \\
\hline 011211 & 2.14 & 4. \pm 1.6 & Sum & $11-12.4$ & $15.6 \pm 6.3$ & $0.25 \pm 0.1$ & $2.2 \pm 0.9$ & R02 & - & - & $0.5-4.4$ \\
\hline
\end{tabular}

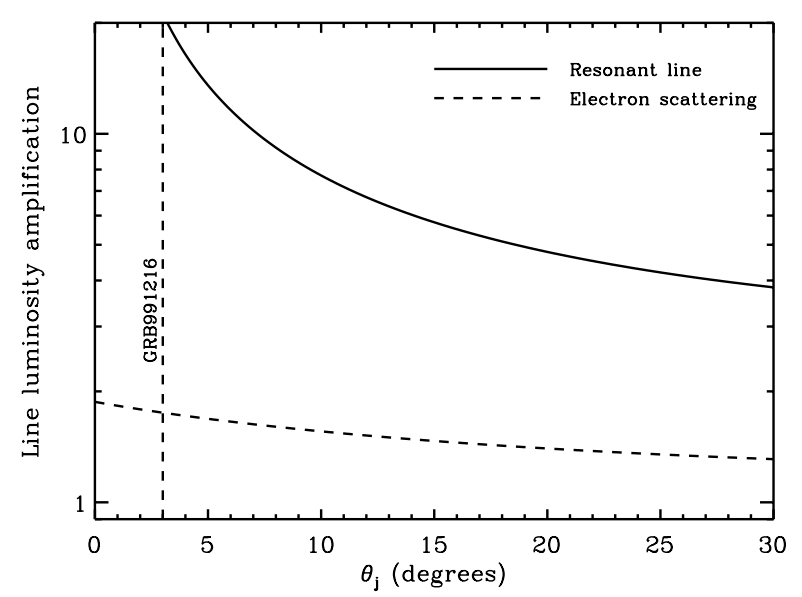

Fig. 1. Amplification of the line luminosity in a funnel after line photons are reprocessed by scattering off the funnel wall.

GRB 011211 the emission lines became undetectable before the end of the $\mathrm{X}$-ray observation. In all cases, despite the range of start times of $\mathrm{X}$-ray observations in different bursts, the line was always visible in the first part of the observations (i.e. there is no indication of line fluxes increasing in time).

\subsection{Line photon collimation}

Even for line photons produced isotropically, it is possible that multiple scatterings off the walls of a funnel produce an effective collimation of the emission, enhancing its observed flux (McLaughlin et al. 2002) by the factor $4 \pi / \Omega_{\text {line }}$ with respect to isotropic emission. Therefore the line photons may be "channeled" into a cone of opening angle comparable to the funnel opening angle. There are however two effects limiting the collimation efficiency. First, photons scattered by the wall of the funnel close to its opening can escape in a direction different from that of the line of sight. These photons will be lost and not redirected towards the observer. Second, for each scattering the line photon energy changes if the scattering is not completely elastic, resulting in the smearing of the line. If the scattering opacity is mainly due to free electrons at a temperature of several keV, the line width after several scatterings (Pozdniakov et al. 1983) is $\sigma_{\epsilon} / \epsilon=\left(2 N_{\mathrm{sc}} k T / m_{\mathrm{e}} c^{2}\right)^{1 / 2}$. The detected lines have widths $\sigma_{\epsilon} / \epsilon \sim 0.1$, from which we can derive $N_{\mathrm{sc}} \sim 3$.

Let us now consider a funnel of height $R$ and opening angle $\theta_{\mathrm{j}}$. A photon scattered at a distance $R z(0 \leq z \leq 1)$ from the funnel opening will have a probability $P_{\mathrm{obs}}=$ $\left(1-\cos \theta_{\mathrm{j}}\right) / 2$ of being scattered towards the observer, and a probability $P_{\text {esc }}(z, \theta)=(1-\cos [\operatorname{atan}(\sin \theta / z)]) / 2$ of being scattered in any direction outside the funnel cone. Assuming that the funnel walls radiate and scatter the line uniformly, the total probability for a photon to exit the funnel is:

$P_{\text {esc }}(\theta)=2 \int_{0}^{1}(1-z) P_{\text {esc }}(z, \theta) \mathrm{d} z$.

In addition we must consider that half of the photons will be scattered in the forward direction, i.e. deeper into the funnel walls rather than towards the open space. These photons will be lost if the main scattering opacity is provided by free electrons. On the other hand, if the considered line is resonant, these photons may be rescattered by ions while still at a small Thomson depth in the walls and redirected towards the open space. The total probability of a photon to be lost is then $P_{\text {lost }}=0.5+P_{\text {esc }}(\theta)$ for electron scattering and $P_{\text {lost }}=P_{\text {esc }}(\theta)$ for a resonant line.

After $N_{\text {sc }}$ scatterings, the total number of photons emitted in the direction of the observer will be enhanced by a factor

$$
\mathcal{F}(\theta)=\sum_{n=0}^{N_{\mathrm{sc}}}\left[1-P_{\text {lost }}(\theta)\right]^{n} ; \quad \lim _{N_{\mathrm{sc}} \rightarrow \infty} \mathcal{F}(\theta)=\frac{1}{P_{\text {lost }}(\theta)}
$$

Figure 1 shows the results of the integral in Eq. (1) as a function of the funnel opening angle $\theta_{\mathrm{j}}$. The solid lines 


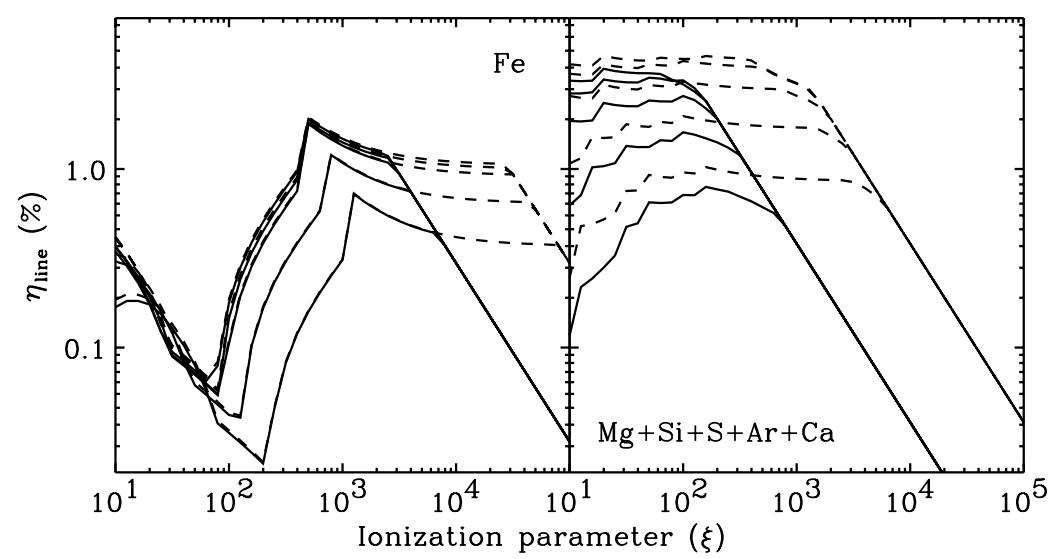

Fig. 2. Efficiency of conversion of continuum [1-30 keV] luminosity into $\mathrm{K}_{\alpha}$ line luminosity as a function of the ionization parameter $\xi \equiv 4 \pi F_{[1-30] \mathrm{keV}} / n$. The continuum is assumed to be a power-law $F(\nu) \propto \nu^{-\alpha}$ within the $[1-30 \mathrm{keV}]$ energy range. The left panel shows iron $K_{\alpha}$ emission, while the right panel shows the sum of $\mathrm{Mg}, \mathrm{Si}, \mathrm{S}, \mathrm{Ar}$ and Ca $\mathrm{K}_{\alpha}$ lines. Different solid lines show the efficiency for $\alpha=0,0.5,1,1.5$ and 2. Dashed lines are obtained with the same parameter set as for solid lines, but with ten times solar iron abundance. The iron peak efficiency $\eta_{\text {line }} \approx 0.02$ is obtained for $0<\alpha<1$. The soft $\mathrm{X}$-ray lines peak efficiency $\eta_{\text {line }} \approx 0.05$ is obtained for $\alpha>1.5$.

show the rather important amplification that a resonant line can achieve (with infinite number of scatterings), while the dashed line shows that, if electron scattering is important, the amplification of the line can be at most a factor of two (the 3 scattering amplification is shown, being very similar to the infinite one).

\section{Energy of the photoionizing continuum}

The efficiency of conversion of the X-ray ionizing continuum into $\mathrm{K}_{\alpha}$ line photons has been recently investigated by Lazzati et al. (2002). They assume that the line is produced by reflection off an optically thick slab of material, illuminated by a power-law continuum. This is the more efficient way to reprocess continuum photons into $\mathrm{K}_{\alpha}$ lines.

Figure 2 shows the result of the efficiency calculations for iron and for the sum of the light elements $\mathrm{Mg}, \mathrm{Si}, \mathrm{S}, \mathrm{Ar}$ and Ca. We explore the effects of different ionizing spectral shapes and metallicity. The reprocessing efficiency for iron cannot be larger than $2 \%$, while the combined light elements, for very small ionization parameters, can reprocess up to $5 \%$ of the continuum into soft X-ray narrow lines.

It is important to note that the iron line is resonant for $10 \lesssim \xi \lesssim 300$ and for $\xi>10^{4}$. In these ranges the efficiency of line production is much smaller due to the Auger disruption of the line (see Ross et al. 1996). This effect compensate for the possible line amplification due to beaming and we will therefore concentrate on nonresonant lines in the following, bearing in mind that similar results also apply to the resonant case.

\section{Limits on the total energy reservoir}

The energy contained in the emission lines sets a lower limit to both the total radiated energy and to the total energy reservoir of the fireball. In fact the line energy is a fraction $\eta_{\text {line }}$ of the illuminating ionizing $[1-30 \mathrm{keV}]$ continuum, which is in turn a fraction $\eta_{x}$ of the energy emitted in $\gamma$-rays during the prompt emission. We take into account that the line photons might be collimated, resulting in an amplification factor $4 \pi / \Omega_{\text {line }}$ with respect to the isotropic case. Considering the efficiency for iron lines (the most commonly detected) the limit to the energy radiated in $\gamma$-rays then reads:

$$
\begin{aligned}
E_{\gamma} & \geq 2 \frac{E_{\text {line }}^{\text {iso }}}{\eta_{x} \eta_{\text {line }}} \frac{\Omega_{\text {line }}}{4 \pi} \\
& =500 E_{\text {line }}^{\text {iso }}\left(\frac{0.1}{\eta_{x}}\right)\left(\frac{0.02}{\eta_{\text {line }}}\right)\left(\frac{\Omega_{\text {line }} / 4 \pi}{0.5}\right)
\end{aligned}
$$

where the factor 2 in the first line of Eq. (3) corresponds to consider two-sided jets with the line emitting material visible on only one side. To find the total energy reservoir in the form of kinetic energy of the fireball we should know the fraction $\eta_{\gamma}$ of it which is radiated. The total fireball energy is then $E=E_{\gamma} / \eta_{\gamma}$. A value $\eta_{\gamma} \sim 0.2$ assumes that the conversion of bulk kinetic energy into radiation is very efficient, and it is the same value used by Frail et al. (2001).

$\eta_{x} \sim 0.05-0.1$ is the value commonly observed during the prompt emission of most bursts: it corresponds to a spectrum $F(\nu) \propto \nu^{0}$ up to $300 \mathrm{keV}$. This implicitly assumes that $\eta_{x}$ is the same inside the entire collimation cone. However, we could have different scenarii, in which the emission at large angles (which is probably the one illuminating the line emitting material) is more $\mathrm{X}$-ray rich than along the jet axis:

- In the model of Mészáros \& Rees (2001), the line emitting material is located in dense clumps inside an hypernova envelope, illuminated by a non-thermal continuum corresponding to the side expansion of the emerging jet. This continuum could peak at the "right" X-ray energies, but since it does not share the time-decay properties of a "standard" afterglow, its non-detection implies that its total energy (i.e. its 
luminosity integrated for the emission time) is less than the total energy contained in the $\mathrm{X}$-ray afterglow. This implies that also in this case $\eta_{x}<0.1$.

- There could be a $\theta$-dependence of the bulk Lorentz factor and the fireball energy, as in the model by Rossi et al. (2002). In this case it is possible that also the emission properties can change with $\theta$, making $\eta_{x}$ a function of $\theta$, possibly reaching $\eta_{x}=1$ at large angles. In this model $\theta_{\mathrm{j}}$ is assumed to be quite large $\left(>25^{\circ}\right)$, implying no or weak collimation of the line photons (i.e. $4 \pi / \Omega_{\text {line }} \rightarrow 1$ ). Furthermore, the energy emitted at large angles is in this model only a fraction of the total, making the lower limit derived by Eq. (3) approximately valid also in this case.

\section{Discussion}

The most severe lower limit to the total energy reservoir is obtained for GRB 991216 if its iron line "lived" for 40 hours (i.e. from soon after the trigger until the end of the Chandra observation). In this case we obtain $E_{\text {line }}=(7.7 \pm 2.3) \times 10^{49} \mathrm{erg}$ corresponding by Eq. (3) to the lower limit $E_{\gamma} \gtrsim 3.8 \times 10^{52}$ erg for the energy radiated in $\gamma$-rays. Instead, if the line was "short lived" and existed only during the 3 hours of the Chandra exposure, we obtain $E_{\text {line }}=(5.8 \pm 2) \times 10^{48} \mathrm{erg}$, corresponding to $E_{\gamma} \gtrsim 2.8 \times 10^{51} \mathrm{erg}$. We then obtain a value which is a factor 4 to 50 greater than what estimated by Frail et al. (2001), which derived a jet opening angle of $\theta_{\mathrm{j}} \sim 2.9^{\circ}$ from the break in the lightcurve 1.2 days after the trigger, and assuming an interstellar medium of density $n=0.1 \mathrm{~cm}^{-3}$. The choice of this somewhat small value for the the circumburst density is in agreement with values derived by modelling broad-band afterglow lightcurves (Panaitescu \& Kumar 2001). Since the calculated fireball energy depends on $n^{1 / 4}$, the lower limit estimated here becomes consistent with achromatic breaks being due to collimated afterglow if the circum-burst density is $n>25 \mathrm{~cm}^{-3}$ (short lived line) or $n>6 \times 10^{5} \mathrm{~cm}^{-3}$ (long lived line). Since the total fireball power depends from the square of the opening angle of the jet, we derive in this case $\theta_{\mathrm{j}}>5.8^{\circ}$ or $\theta_{\mathrm{j}}>20^{\circ}$ for the short or long lived line, respectively.

For GRB 970508 we derive $E_{\gamma} \gtrsim 10^{52} \mathrm{erg}$, a factor $\sim 50$ above the estimate of Frail et al. (2001) for the same burst. This is particularly puzzling, since for this burst the radio data (the source was followed for 450 days) can be used to calculate the energy reservoir in an independent way, which yields $E \sim 5 \times 10^{50}$ erg (Frail et al. 2000), consistent with the value of Frail et al. (2001), but rather inconsistent with the iron line data. Wijers \& Galama (1999), fitting the afterglow spectrum of this burst (from radio to $\mathrm{X}$-rays), derived a somewhat larger value $(\mathrm{d} E / \mathrm{d} \Omega) \sim 3 \times 10^{52} \mathrm{ergs} / 4 \pi \mathrm{sr}$, corresponding, for $\theta_{\mathrm{j}}=16.7$ degrees, to $E \sim 1.6 \times 10^{52} \mathrm{erg}$ (two jets). Again, an increase of the circumburst density to $n \sim 6 \times 10^{5} \mathrm{~cm}^{-3}$ makes our limit consistent with the value of the fireball energy derived by the presence of the achromatic break in the afterglow lightcurve.
In GRB 011211 we only see emission line for elements lighter than iron (Reeves et al. 2002; see Lazzati et al. 2002 for discussion), and correspondingly used $\eta_{\text {line }}=0.05$ to derive the lower limits $E_{\gamma} \gtrsim 5 \times 10^{50}$ erg and $E_{\gamma} \gtrsim$ $4.4 \times 10^{51} \mathrm{erg}$ for a short or long lived line, respectively.

\section{Conclusions}

If real, the line emission features observed in the X-ray afterglow of GRBs are very luminous, and pose strong limits to the total energetics of GRBs. Since these limits are almost unaffected by the abundance of metals, emission mechanisms and collimation of the illuminating continuum, we consider them to be quite robust. If we want to reconcile these limits with the estimates of the jet opening angle and total energy reservoir derived through the achromatic breaks of the afterglow lightcurve, we are led to consider much larger densities of the material surrounding the bursts, in contrast with the results from broad band spectral fitting (e.g. Panaitescu \& Kumar 2001).

We conclude by stressing the importance of the X-ray follow-up observations, such as the one already possible with Chandra and XMM-Newton, and the future one which can be performed by the Swift satellite. Observing emission features in the $\mathrm{X}$-ray spectra can immediately set powerful limits to the total energetics, while observations of the time duration of the line (and its possible variability behavior) can shed information on the illuminator and on the geometry of the line emitting material.

Acknowledgements. GG, DL and ER thank the MIUR for the No. 2001-02-43 grant. ER thanks the Isaac Newton and PPARC scholarships for financial support.

\section{References}

Antonelli, L. A., Piro, L., Vietri, M., et al. 2000, ApJ, 545, L39 Frail, D. A., Waxman, E., \& Kulkarni, S. R. 2000, ApJ, 537, 191

Frail, D. A., Kulkarni, S. R., Sari, R., et al. 2001, ApJ, 562, L55

Lazzati, D., Ghisellini, G., Amati, L., et al. 2001, ApJ, 556, 471

Lazzati, D., Ramirez-Ruiz, E., \& Rees, M. J. 2002, ApJ, submitted [astro-ph/0204319]

McLaughlin, G. C., Wijers, R. A. M. J., Brown, G. E., \& Bethe, H. A. 2002, ApJ, submitted [astro-ph/0110614]

Mészáros, P., \& Rees, M. J. 2001, ApJ, 556, L37

Panaitescu, A., \& Kumar, P. 2001, ApJ, 554, 667

Piro, L., Costa, E., Feroci, M., et al. 1999, ApJ, 514, L73

Piro, L., Garmire, G., Garcia, M., et al. 2000, Science, 290, 955

Pozdniakov, L. A., Sobol, I. M., \& Sunyaev, R. A. 1983, Astrophys. and Space Phys. Rev., 2, 189

Reeves, J. N., Watson, D., Osborne, J. P., et al. 2002, Nature, 416,512

Rhoads, J. E. 1999, ApJ, 525, 737

Ross, R. R., Fabian, A. C., \& Brandt, W. N. 1996, MNRAS, 278,1082

Rossi, E., Lazzati, D., \& Rees, M. J. 2002, MNRAS, 332, 945

Wijers, R. A. M. J., \& Galama, T. J. 1999, ApJ, 523, 177

Yoshida, A., Namiki, M., Otani, C., et al. 1999, A\&AS, 138, 433 